Aus der Chirurgischen Universitätsklinik und -poliklinik in Berlin. (Direktor: Geh. Med.-Rat Prof. Dr. Bier.)

\title{
Beitrag zur operativen Behandlung der Bechterewschen Krankheit.
}

\author{
Von Dr. Karl Stern, Volontär-Assistent.
}

In einer neueren Arbeit aus der Krausschen Klinik hat Plehn mit Nachdruck darauf hingewiesen, daß die Kranken mit chronischer Steifigkeit der Wirbelsäule nicht an ihrer eigentlichen Krankheit zugrunde gehen, sondern daß die schließliche Todesursache bedingt ist durch die Folgezuständo der Wirbelsäulen- und Thoraxankylose, nämlich durch ,Inkompetenz des Kreislaufs und ver allem durch Insuffizienz der Atmung".

Die schönen Untersuchungen Plehns lassen eine klare Vorstellung gewinnen von den gefahrdrohenden, einschneidenden Veränderungen hinsichtlich der respiratorischen Verhältniss , die bei Kranken mit Bechterewscher oder Strü mpell-Mariescher Krankheit sich finden.

Sie beziehen sich in der Hauptsache einmal auf den Nachweis des sehr verminderten Gehalts der Lungen an Residualluft infolge der ständigen Exspirationsstellung des Thorax, dann auf den ungemein geringen Sauerstoffgehalt des venösen Bluts, ein Untersuchungsergebnis, das zur Geniige dartut, in wie hohem Maße der Organismus dieser Kranken den Sauerstoff ausnutzen muß, um lebensfälig zu bleiben.

Die geringe Sanerstoffzufuhr, die der Organismus erfälırt, hat ihren Grund vor allem darin, daß die Atnung keine thorakale, sondern eine abdominale, nämlich eine reine Zwerchfellatmung ist und daß die Atemfrequenz, un das Zwerchfell zu schonen, in solchem Maße eine Verringerung zu erfahren pflegt, daß ein Sinken der Frequenz auf zehn Atemzïge in der Minute nichts Ungewöhnliches bedeutet.

Durch die Untersuchungen Plehns sind der Therapic bei der in Rede stehenden Erkrankung neue Wege gewiesen, und ihre Behandlung, der man mit den bisher angewandten internen, physikalischen und diätetischen Maßnahmen machtlos gegenüberstand, ist gewissermaßen chirurgisch geworden. Denn es gilt, durch Mobilisierung des Thorax eine Aenderung der respiratorischen Verhältnisse herbeizuführen und durch völlige oder wenigstens teilweise Wiederherstellung der thorakalen Atmung der Indicatio vitalis zu genügen.

Auf Grund dieser Ueberlegungen hat Klapp cinen Patienten mit Bechterewscher Krankbeit einer operativen Behandlung unterzogen, und der durch die Oprration erziclte Erfolg rechtfertigt es, den Fall mitzuteilen.

Die Krankengeschichte ist folgende:

A. Sch., 50 Jahre, Diener. Aufgenommen 9. März 1911 ; entlasscen 15. April 1911. Anamnese: Vor 25 Jahren Gonorrhoe; seit sechs Jahren bemerkt er eine Versteifung der oberen Wirbelsäule, die immer meh. zunahm; seit der gleichen Zeit wird die Haltung immer gebeugter. Schmerzen waren zeitweise vorhanden und traten besonders bei Wetterveränderungen auf. Seit drei Jahren hat er Beklemmungen auf der Brust und infolgedessen Angstzustände, die ihn nötigen, nächtelang das Bett zu meiden. Von einer gleichen Erkrankung in der Familie ist ihm nicht bekannt. Getrunken hat er nie. Jetzt bestehen seit Monaten Schmer zen im Hinterkopf, Nacken und Rücken. Er kann nur nit offenem Mund liegen und schnarcht deshalb naehts sehr stark.

Status: Mittelgroßer, mäßig gut genährter Mann. Herz, Lungen ohne Besonderheiten. Der Brustkorb ist bei der Atmung unbeweglich die respiratorische Exkursion beträgt ïber den Mamillen gemessen 1 c'm; das Abdomen wird in scinen oberen Partien bei der Exspiration sehr stark eingezogen (Zwerchfellatmung). Im Liegen kann Patient nur nit offenem Munde atmen, die Interkostalräume sind selı eng, die Rippen steil abfallend. Die Wirbelsäule ist im Bereich der Hals- und Brustwirbelsäule stark kyphotisch und unbeweglich; die Kyphose ist bogenförmig, jedoch nicht rein bogenförmig, vielmehr treten die beiden untersten Hals- und der oberste Brustwirbel gibbusartig hervor. Der Kopf ist stark nach vorn gerückt und der Brust genähert. Die Sprache ist leise, abgerissen, mühsan. Der Gang langsanı, breitbeinig, schwerfällig mi kurzen, ängstlichen Schritten. Aufrichten aus sitzender l'osition geschieh ${ }^{\dagger}$ 
mit Zuhilfenahme der Hände; ebenso das Aufrichten aus gebückter Stellung. Die Wirbelsäule ist nicht druckempfindlich; sämtliche Extremitätengelenke sind frei. Die Atemfrequenz ist auffallend gering.

25. März Operation (Professor Klapp): Morphium, Atropin, Aether.

Schnitt beiderseits längs der Rippenknorpelknochengrenze, von del' Clavionla bis zum Epigastrinm. Die Schnitte wenden sich nach unten zll im Bogen medianwärts.

Von der 2.-8. Rippe werden $2-5 \mathrm{~cm}$ lange Knorpelstücke subperiostal reseziert, und das steliengebliebene Periost wird init dem Paquelin verschorft. Die Rippenknorpel sind opak verfärbt, vielfach verdickt und mit dem Perichondrium abnorm stark verwachsen. Nach Durchtrennung der linken zweiten Rippe schieben sich die Enden $1 / 2 \mathrm{~cm}$ iibereinandel lind nach Dilchtrennung aller Rippen 1 cm. Rechts ist die Uebereinanderlager'ung der Rippenenden nicht so ausgesprochen. Pleura wird nicht eröfinet. Hautnaht.

26. März. Patient gibt bald nach dem Aufwachen an, daß er besser Atenl bekommt als vol der Operation. 15. April entlassen. Schläft gut, schnarcht nicht mehr; keine Beklemmungen mehr. Keine Sclimerzen im Hinterkopf, Nacken oder Rücken.

Die Nachbehandlung bestand in aktiver 'Thoraxgymnastik dulch Kriechïibu ngen.

Nachunter'suchung am 23. September 1911. Patient liat die Kriech. iibungen zll Hause fleißig fortgesetzt. Seit del Operation keine Selımerzen mehr, keine Beklemmungen mehr anf der Brllst; kann wieder gerade gehen. "Seine Bekannten erkennen ihn wegen seinel geraden Haltung nicht wieder." Kann weit und schnell gehen, gut Treppen stcigen. Kyphose geschwunden, ebenso der Gibbus; der Kopf wird gerade gelialten; kann nach hinten gebeugt werden; deutliche thorakale Atmung. Atemcxkursion $4_{1 / 2}^{1 / 2} \mathrm{~cm}$; (Exspiration $81, \frac{1}{2} \mathrm{~cm}$, Inspiration $86 \mathrm{~cm}$ ). Die Atınung inl Liegen geschieht mit geschlossenem Munde. Zwischenrippenräunıc normal weit, Atemfrequenz: 20 Atemzüge in der Minnte. Sprache: frei, laut, zusammenhängend. Gang: elastisch, gloße, schnelle Schritte. Aufstehen aus sitzendel Position: behend und leicht. Dic r'espiratorischen Exkırsionen gehen an derResektionsstelle der Rippen vor sich ; die Rippenenden haben sich iibereinandergeschoben derart, daß das mediale Ende oberhalb des lateralen steht. Bei der Atming schiebt sich das ganze Mittelstiick, Sternım und inediale Rippenfragmente, nach anfwärts.

Der respiratorische Stillstand des Brustkorbs, als cin fast konstantes Symptom bei gleichzeitiger Ausbildung cines rein abdominalen Atmungstypus, findet sich schon bei Bechterew vermerkt, der gleichzeitig die Verninderung des Tiefendurchmessers des Thorax hervorhebt. Auch Pierre Marie und seine Schïller erwähnen den respiratorischen Stillstand des Brustkorbs bui gleichzeitiger Abflachung desselben. Dabei lenkt Pierre Marie ausdriicklich die Aufmerksankeit dalauf, dal die Rippen an dic Wirbelsäule fixiert seien.

Erst Plehn aber rïckt mit Recht die Fixationsstellung der Rippenwirbclgelenke in den Vordergrund des Interesses und macht sie zum Gegenstand einer besonderen Abliandlung, inden er auf die Fixation der Rippenwirbelgelenke das Fohken der thorakalen Atmung und die Ausbildıng der 7worchfellatmung \%urückführt.

Es bezweckt nänılich der Kranke durch dic abdominalc Atmung b\%w. durch die Rubigstellung seines Brustkorbs rinc Entlastung der schmerzhaften Rippenwirbelgolenke, die anfang; durch Muskelkontraktur, später durch knöchernc; Fixation in üußerster Exspirationsstellung fixiert gelialten werden, da sie so am moisten entlastet sind.

Dic: Flage, auf welcher Trsache; die Fixation des 'Thorax beruht, ist für dic krwägung von dem Nutzen der opcrativen Mobilisation von prinzipiclior Bedeutung, und ilı* Beantwortung geschieht kcineswegs eindentig in dem sinne Plehns.

So verticten einige Autoren den Standpunkt, daß3 der rospiratorische Atemstillstand bedingt sei durch Atrophic der Respirations- resp. respiratorischen Hilfsmuskeln.

Auch Bccli terew liat, gestiitzt anf einen Sektionsbefund, in seinen Darlegungell über die nach ihm benannte Erkrankıng dieser Anschaulıng Ausdl'lıek verliehen, in der Annahme, daß das Primäre der Erklankung eine Affektion der Pia sei, die ihrerseits zll Konıpression der Wulzeln r11 der Eintrittsstelle fiihrt, und diese Wurzeldegeneration bezeichnet or ils dic Ursache der Paresen dor Muskelgruppen des Thorax.

Der Sektionsbefund Bechterews hat dilrel die übrigen vorliegenden pathologisch-anatomischen Untersichungsergobnisse keine $\mathrm{Bc}$ stätiglıng elfahren könnell. Uebereinstimmend wird vielmehr als das Halıptsäehliche des pathologisch-anatomisclien Vorgangs eine Verknöclerlıng dor Bänder der Wirbelsäule und Ossifikation und Verwachsung der (relcnkc zwischen Rippen und Wirbelsäule angesehen.

Würde: die Anschauung Beohtercws \%u Recht bostehicn, dal3 diø sekundäre: Muskrelatrophic: dic Ursache des respjirato- rischen Thoraxstillstandes sei, dann wïrde eine operative Mobilisierung einen nur sehr geringen oder gar negativen therapeutischen Effekt erzielen. Denn mit der Resektion von Rippenknorpelstïcken oder der Rippenwirbelgelenke wird zwar eine Mobilisation der Rippen erzielt, allein das Fortbestehen der Insuffizienz der Respirations- und respiratorischen Hilfsmuskeln könnte alsdann den thorakalen Atmungsstillstand nicht beseitigen.

Allein es läßt der Effekt, den die Klappsche Operation cimielt hat, den Schluß zu, daß der Atemstillstand des Thorax nicht bedingt ist durch die Atrophie der entsprechend. $n$ Muskeln. Denn es konnte ein halbes Jahr nach der Operation eine rcoht erhebliche Zunahne der Muskulatur des Rückens und der Interkostalräume konstatiert werden, ein Beweis dafür, daß mit der Mobilisierung des Brustkorbs auch dic: Muskeln wieder ihre Tätigkeit aufzunehnen inıstande sind und daß die Atiophic lediglich als eine Aktivitätsatıophic inzusehen ist.

Klappl hat nicht, wie Plchn vorgeschlagen, dic Ripponwirbelgelenke resc\%iert, vielnelor bis $5 \mathrm{~cm}$ lange Stïckc aus den Rippenknorpeln subperichondral lorausgeschnitten und. das Porichondrium nit den Paquelin verödet. Der Erfolg dieser Oporation war', wie aus der: Krankengeschichte ersichtlich ist, gan\% wesentlich. Vor allen ist durch den Eingriff der fast röllig fixierte Brustkorb wieder soweit nobilisiert worden, dalo dic Ateniexkursionen, dic vor dem Fingriff ctwas nehr als einen Kentimetcr betrugen, boi dor Nachuntersuchung nach einem halben Jalire (.inc: Erlöblung auf nahe:u fünf $/ /$ entinieter $\cdot \cdot r$ fahren hattr.n.

Es lïßt sich leicht und deutlich r.kennen, daß die Atencxkursionen d.es Thorax in den nougebildeten Rippengclenkcn an der R:sektionsstclle ror sich gehon; dabei haben sich dic Rippencnden \%umeist ïbercinander geschoben, derart dal. das mediale oberhalb des lateralen zu stehen kommt, und nur an cinigen wenigen Sitellon bostchlt noch eine Diastaso von etwa cinc.m Kentimeter.

Die Besserung der Atmung wird von don P'atienten solbst iiberaus dankbar anerkannt; sc.it der Operation lat $\mathrm{cr}$ seinc Nachtrulı; wicder, dic Sprach, dic vorker abgebrochen und nühsan war, ist wieder fließend und leicht. und körperlicho Bewegungen, wie langes und schnclles Gehen, sogal Laufen und rilige:s 'Treppensteig( $\cdot \mathbf{n}$, machon keino Kurkatmigkeit. Auch schläft 'r jetzt nit geschlossenen Mundc; und das gänzliche Aufhören des frühor sehr lauten Schnarchens wird von der Unigebung des Pationten dankbal empfundon. Dis: Atemfrequen\%, dic vor denı lingriff vernindert war, ist

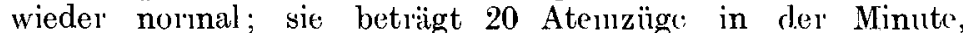
und dic infolge der :xspiratorischen Fixationsstellung ung:ulcin vorengten Zwischenrippentäume weisen wieder die üblicho Weite auf. Ebenso haben die Parästhesien, die als Gïrtclgc fühl und oft unerträgliche Schnerzen in Nacken und Kopf sich äuBerten, alsbald nach der Operation nachgelassen, und scit Monaten sind sie völlig geschwunden.

Diese Parästhesion, dif; eine fast st:̈ndige Begleiterscheinung der in Rede steblenden Erkrankung sind, weid.n all-

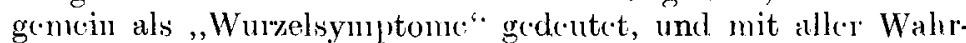
scheinlichkeit sind sic hedingt dureh dic verinderten statischon Verliältnisse der Wirbels:̈ulc.

Die Affektion der. Wirbchsiule, dic stets in Vordergrunde de. Erkrankung steht und dic walırschcinlich als das Prinäre anzuselien ist, wailuend die Vorsteifung der Rippenwirbelgelenkc erst sckundär erfolgt (Kla pl), äußert sich in dcr Ausbildung einer völligen oder fast völligen Ankylose und in einc' Veränderung der normalen Krüunulung der Wirbelsäiulc zu Kyphose.

In ınscren Falle war dic Ankylose cine völlige, und dic Kyphose, wic gewöhnlich dic: oberste: Brust- und dic Halswirbolsälule betreffend, war so stark alsgebildet, daß dis Kinn dem Brustbein fast auflag und in Riickenlagc do ${ }^{*} \mathrm{Kopf}$ des Patienten die Unterlage nicht erreichte.

Anch dic Besserung, wolche diese Wirbelsäiul'nveräindclung durel die Klappsche Operation erfaliren lat, ist als erlecblich zu beztichnon. I)as Kinn wird völlig frei getragen; auch kann dor Kopf nach den Nacken lin gobougt werdon, 
und wenn auch die Beugung nicht in dem Maße vor sich geht wie bei einem völlig Gesunden, so kann der Patient doch wieder „ohne Anstrengung den Himmel sehen".

Ob die Resektion der Rippenwirbelgelenke allein imstando sein würde, auch auf die Kyphose bessernd oder beseitigend zu wirken, bliebe dahingestellt. Gerade die Beseitigung der Kyphose, die ein ganz erhebliches Moment für die Wiederherstellung der Thoraxatmung bedeutet, ist erzielt worden durch die Beweglichmachung des Mittelstücks des Brustkorbs (Brustbein plus mediale Rippensegmente).

Eine sehr augenfällige Veränderung zum Besseren bildet für den, der den Kranken vor und nach d.cr Operation zu sehen Gelegenheit hatte, das Verschwinden des gibbusartigen Vorsprungs im Bereiche der obersten Brust- und unteren Halswirbel. Es beweist dies? Tatsache am besten ein Zurïckgehen nicht nur der Kyphose, sondern auch der Ankylose, denn die Rippenresektion und die Kräftigung der Rücken- und Nackenmuskulatur allein wäre nicht imstande gewesen, diesen Gibbus zu bassitigen, ohne ein gewisses Maß von Beweglichkeit in den betailigten Abschnitten der Wirbelsäule.

An sich ist der gibbusartige Vorsprung dreier Wirbe I an der Grenze von Hals- und. Brustwirbelsäule inmitten der sonst bogenförmigen Kyphose etwas Ungewöhnliches bei der Bechterewschen Krankheit, da übereinstimmend in allen Fällen die Kyphose als eine ,nur bogenförmige ohne stärkeres Hervortreten eines oder mehrerer Wirbel geschildert wird".

Hinsichtlich der Einordnung in eine der beiden Hauptgruppen von Wirbelsäulenversteifung ist zu betonen, daß es sich in unserem Falle um eine reine Form vom Bcchteru schen Typus handelt. Als das wesentliche Unterscheidungsmerkmal der boiden Formen gilt dic Beteiligung oder das Freibleiben dcr ,Wurzelgelenke". Von Pierre Marie stammt daher die Bezeichnung: Spondylose rhizomalique; allein schon seine Schüler beschreiben Fälle, in denen nicht nur die ,Wurzelgelenke" (Hüft- und Schultergelenke) sich ergriffen fanden, sondern anch Knie-, Kiefer- und Fußgelenke.

Spätere Mitteilungen weiterer Fälle haben dann gezeigt, daß der noch von den Schülern Maries aufrecht erhaltene Satz, daß die kleinen Extremitätengelenke frei bleiben, in dieser entschiedencn Form nicht zu Recht besteht.

Allerdings trifft auch die Benennung Bechterews, der die von ihm 1893 zuerst beschriebene Erkrankung als ,,Spondylose hérédotraumatique " bozeichnet, in unserem Falle nicht ganz zu, soweit es sich um die durch das Beiwort gekennzeichnete Aetiologie handelt. Vielmehr weist die Anamnese unsores Patienten darauf hin, daß man in Erkältungssschädlichkeiten und Durchnässungen, denen der Patient als ,Diener" auf Reisen und Jagden sehr viel ausgesetzt war, dic eigentliche Ursache des Prozesses zu suchen hat. Lues, die Bechterew, oder ein Trauma, das Pierre Marie als charakteristisches ätiologisches Moment ansieht, kommen nicht in $\mathrm{Be}$ tracht.

„Die lang dauernden und anstrengenden Eisenbahnfahrten" - wie es Bechterew in zwei Fällen tut - in Sinne eines Traumas zu deuten, orscheint nicht angebracht. Zwar rechnet Pierre Marie die auf gonorrhoischer Basis entstandenen Fälle seinem Symptomenbild zu; allein es lifgt bsi unserem Patienten die gonorrhcische Affektion 25 Jahr., d. h. zu lange Zeit zurïck, um den Prozeß noch als, gonorrhoische Arthritis" anzuschuldigen.

Der therapeutische Effekt der Klappschen Operation, die einen schwer leidenden Menschen wieder Lebensfähigkeit verschafft und Lebensfrende zurückgegeben hat, fordert zur Nachprüfung dieses ncuen Weges in der Behandlung der Wirbelsäulenankylosen und ihrer Folgezustände auf. Ob die Resektion der Rippenwirbelgelenke, die Plehn vorgeschlagen hat, ein noch besseres Resultat ergibt, muB die Erfahrung lehren. Jedenfalls wird Klapp in Zukunft in gegebenen Fïllen außer der Rippenknorpelresektion gleichzeitig die Resektion der entsprechenden Rippenwirbelgelenke ausführen.

Literatur: Heiligenthal, Zentraiblatt für die Grenzgebiete 1900. - Plehn, Deutsche medizinische Wochenschrift 19l1, No. 30. - Anschütz, Glaser, Mitteilungen aus den Grenzgebieten, Bd. 8, - Focken, Mitteilungen aus den Grenzgebieten, Bd. 11. - Ehrhardt, Mitteilungen aus den Grenzgebieten, Bd. 14. 